\title{
Special issue on virtual and mixed reality in culture and heritage
}

\author{
Damianos Gavalas $^{1} \cdot$ Stella Sylaiou ${ }^{2} \cdot$ Vlasios Kasapakis $^{3}$ - Elena Dzardanova ${ }^{1}$
}

Published online: 1 February 2020

(C) Springer-Verlag London Ltd., part of Springer Nature 2020

\section{Introduction and motivation}

The rapid advances in Information and Communication Technologies (ICT) has profoundly impacted the cultural industry, among others. Cultural institutions are commonly on the forefront of technological innovations, persistently exploring compelling ways to address new audiences and introduce novel means of interaction with cultural assets, thus transforming, enriching and enhancing the perceived cultural experience. The potentials of the new tools trigger new transdisciplinary perspectives for presentation, representation, management, interpretation, personalization and effective dissemination of information concerning cultural heritage and art. The emerging technological instruments are exploited to enable enhanced access not only in 'brick-and-mortar', but also in virtual cultural exhibitions.

The recent explosion of the immersive technologies (Virtual Reality - VR and Mixed Reality - MR) offers unprecedented opportunities for learning, communication and entertainment in cultural spaces. VR/MR may play a key role in the presentation of the past and of polysemic cultural practices which can allow new sensations and meanings to arise. Among others, VR/MR technologies enable the exploration and interaction with important cultural artifacts, whilst in the

Damianos Gavalas is the Lead guest editor.
Damianos Gavalas
dgavalas@aegean.gr
Stella Sylaiou
sylaiou@gmail.com
Vlasios Kasapakis
v.kasapakis@aegean.gr
Elena Dzardanova
lena@aegean.gr
1 University of the Aegean, Syros, Greece
2 Hellenic Open University, Patras, Greece
3 University of the Aegean, Mytilene, Greece

physical space or immersed in a virtual environment; incorporate stimulating forms of storytelling to further entertain and engage visitors; allow the combination of exhibits physically distant from each other, or the exhibition of objects that have no physical presence and are born digital; provide valuable help for the digital preservation of heritage sites and cultural collections, research and communication for education, entertainment and tourism promotion. The affordances of immersive technologies urge cultural organizations to rethink, reimagine and perhaps reshape the very concept of museums and heritage sites.

The motivation behind this special issue is to solicit highquality articles on all aspects pertinent to Virtual and Mixed Reality in Culture and Heritage and outline the state of the art in this exciting area of research.

\section{Submissions, review process, summary of contributions}

The special issue of the Springer's Personal and Ubiquitous Computing on "Virtual and Mixed Reality in Culture and Heritage" has attracted numerous submissions by authors affiliated with institutions in Belgium, Czech Republic, Cyprus, Germany, Greece, Israel, Malaysia, Spain, Taiwan and United Kingdom. Following a rigorous review process, 6 outstanding papers have been finally selected for inclusion in the special issue. Each paper received three reviews from independent experts. The accepted papers cover a wide spectrum of research topics in the broader area of the special issue.

The first paper, entitled "A Hybrid Augmented Reality Guide for Underwater Cultural Heritage Sites" (by J. Čejka, A. Zsíros and F. Liarokapis), presents a novel augmented reality guide for divers to present ancient lost buildings at underwater archeological sites. The prototype system runs on a smartphone sealed in a waterproof case and uses a hybrid approach (markers and inertial sensors) to localize the diver. The application has been experimentally evaluated at an underwater archeological site in Italy, demonstrating the 
potential of alike guides to enhance user experience in underwater archeological sites.

The second article, titled "Avatars as Storytellers: Affective Narratives in Virtual Museums" (co-authored by S. Sylaiou, V. Kasapakis, D. Gavalas and E. Dzardanova), investigates the persuasiveness and overall emotional impact of various types of virtual guides in virtual museum environments. Three avatars with different professional and social characteristics (personifying a curator, a guard and a visitor, respectively) have been employed as storytellers introducing participants to the emotive story behind a historical sculpture. The key objective of the paper is to inform the design of avatars-as-storytellers in virtual museums on the basis of their affective potential, based on the interpretation of results derived from a user study. The paper reports on the emotional responses of a test group which have been correlated to a range of factors, namely, the role acted by the virtual storytellers, the subjects' own stance on cultural heritage, gender and predispositions towards the sense of presence and affective responses.

The third paper, entitled "Design Methodology for $360^{\circ}$ Immersive Video Applications: The Case Study of a Cultural Heritage Virtual Tour" (contributed by L. Argyriou, D. Economou and V. Bouki), introduces a methodological categorization of design aspects pertinent to the development of $360^{\circ}$ immersive video applications (narrative design, rendering of virtual scenes, role of actors, navigation means, gamification elements). The article then presents a testbed application (immersive interactive virtual tour of the city of Rethymno, Greece), which serves the purpose of testing the attitude of users with respect to these design aspects. Based on the analysis of the results of the user study, the article presents a set of design guidelines for the implementation of $360^{\circ}$ immersive video virtual tours.

The fourth paper, entitled "The Effect of Immersion Towards Place Presence in Virtual Heritage Environments" (co-authored by I. Ghani, A. Rafi and P. Woods), presents the methods and findings from a qualitative experiment designed to analyze user's real-time feedbacks from three levels of immersive systems (non-immersive, semi-immersive and fully-immersive). The experiment aims to measure the significance of virtual presence in contributing towards user's sense of place experience in a virtual heritage environment. The paper reports on the results and findings of the experiment and then discusses the effectiveness of virtual heritage environments in knowledge dissemination and cultural heritage learning experience.
The fifth paper, entitled "Enhancing Cultural Heritage Outdoor Experience with Augmented-Reality Smart Glasses " (co-authored by E. Litvak and T. Kuflik), presents a mobile augmented reality guide based on smart glasses, which serves as a testbed for exploring the potential of this medium for enhancing visitor experience at outdoor cultural heritage sites. The paper discusses the design considerations and implementation details of the augmented reality smart glasses-based guide which provides visitors with context-aware information relevant to points of interest within their field of view. The guide prototype has been compared against a 'conventional' smartphone-based guide baseline. The results reported from lab experiments and field trials demonstrate the positive attitude of visitors towards the smart glasses-based guide for exploring heritage sites, but also highlight several critical challenges which must be carefully addressed by developers of similar systems.

The last paper, entitled "Culture 4 All: AccessibilityEnabled Cultural Experiences through Immersive VR360 content" (by M. Montagud, P. Orero and A. Matamala), investigates the largely unexplored aspect of accessibility in VR360 videos. This article firstly reviews the needs, challenges and limitations for making VR360 experiences accessible. Based on these facts, an end-to-end platform to efficiently integrate accessibility services within VR360 content is presented. The platform encompasses all steps from media authoring to media consumption, emphasizing on the accessibility-enabled VR360 player, being the main end user interaction interface. The paper then investigates the presentation modes for the supported access services (like subtitling, audio description and sign language), interaction modalities and personalization features supported by the player.

Acknowledgments We would like to thank the authors of all the submitted papers for considering our special issue and the Personal and Ubiquitous Computing journal as a potential publication venue for their research results. We would like to especially thank the authors of the accepted papers for their effort in revising and improving their work occasionally several times-in response to reviewer comments.

This organization of this special issue has been co-financed by Greece and the European Union (European Social Fund- ESF) through the Operational Programme "Human Resources Development, Education and Lifelong Learning 2014-2020" in the context of the project "Social Interaction in Virtual Reality Environments" (MIS 5004223).

Publisher's note Springer Nature remains neutral with regard to jurisdictional claims in published maps and institutional affiliations. 